I. Del Prete, Dipartimento di Matematica e Applicazioni, Facoltà di Ingegneria, Università degli Studi di Napoli, Via Claudio 21, 80125 Napoli, Italy. e-mail: delprete@cds.unina.it

M. Di Iorio, Dipartimento di Matematica e Applicazioni, Facoltà di Ingegneria, Università degli Studi di Napoli, Via Claudio 21, 80125 Napoli, Italy. e-mail: diiorio@cds.unina.it

S. Naimpally, 121 Medhurst Drive, Nepean, Ontario, K2G 4J9 Canada. e-mail: snaimpal@math.carleton.ca

\title{
ESSENTIAL FIXED POINTS OF FUNCTIONS AND MULTIFUNCTIONS ${ }^{\dagger}$
}

\begin{abstract}
Suppose $(X, d)$ is a compact metric space with the fixed point property and $\mathbb{C}$ the family of all continuous self maps on $X$ with the topology of uniform convergence. A fixed point $p$ of $f \in \mathbb{C}$ is said to be essential if functions near $f$ have fixed points near $p$. A function which has all of its fixed points essential is called an essential map. Fort [4] proved that the set of essential maps is residual in $\mathbb{C}$ and yet the only known examples of essential maps are those with only one fixed point. In this paper working in $[0,1]$, we first characterize essential fixed points and prove some simple results concerning them. Then we characterize essential maps and give algorithms to construct them. We then study essential components introduced by Kinoshita [8]. Next we consider essential fixed points of multifunctions in which case results differ considerably from the case of single valued functions. This also leads us to a study of selections. We conclude with a study of essential fixed points of non expansive functions in Banach spaces. All along we provide examples to illustrate the concepts and their limitations. Our results throw light on what is already known and takes the subject further. Unsolved problems are also mentioned.
\end{abstract}

Key Words: fixed point, essential fixed point, map, essential map, non expansive map, multifunction, selection

Mathematical Reviews subject classification: Primary 54H25, 47H09, 47H10; Secondary 54B20, 54C35

Received by the editors April 2, 1998

$\dagger$ Work partially supported by CNR and MURST.

*This author is grateful to the first two for their kind hospitality. 


\section{Introduction}

The concept of an essential fixed point was introduced by M. K. Fort [4]. It is a kind of stability and has been successfully used in studying Nash Equilibrium points in Game Theory by Jiang Jia-He [7]. We believe that it can be used in studying stability in Differential equations as well as in Dynamics; in this connection see Bruckner's paper [3]. Fort showed that, under suitable conditions, functions that have all fixed points essential (essential maps) form a residual set. In the general case of a metric space with fixed point property, Fort asked for examples of essential maps. But in spite of forming a residual set, the only known examples of essential maps in the literature are those with only one fixed point. In this paper, we completely classify fixed points of self maps in $[0,1]$ and provide techniques to construct essential maps. We also characterize them. Similar problems of characterizing essential fixed points and essential maps on $[0,1]^{n}, n>1$ as well as on the well known spaces with fixed point property, such as compact convex subsets of a Banach space, are still open. Kinoshita [8] introduced the concept of an essential component. This is a non trivial concept since a function may not have essential fixed point and yet have an essential component. We characterize essential components and provide a technique for the construction of maps with essential components. We then study essential fixed point of multifunctions. Here the situation is quite different from that of single valued maps and thus making it interesting. Our study continues with comparisons of essential points of selections with those of multifunctions. The relationship between single valued selections and multifunctions is intriguing and there are some surprising results here. Finally, we study essential fixed points of non-expansive self maps on Banach spaces. Under suitable conditions such maps have the fixed point property, but can have more than one fixed point. We show that essential non-expansive maps are those with only one fixed point.

\section{Preliminary Definitions and Results}

Let $(X, d)$ be a compact metric space with the fixed point property, that is every continuous function from $X$ to $X$ has a fixed point. Let $C L(X)$ denote the family of nonempty closed (compact) subsets of $X$. We recall that if $A$ and $B$ belong to $C L(X)$

$$
\begin{gathered}
d(x, A)=\inf \{d(x, y): y \in A\} \\
d_{H}(A, B)=\max \left\{\sup _{x \in B} d(x, A), \sup _{y \in A} d(y, B)\right\} .
\end{gathered}
$$


We denote by $\mathbb{C}$ the set of continuous single valued functions on $X$ to $X$, by $\mathbb{M}$ the set of multivalued continuous functions from $X$ to $C L(X)$, by $\mathbb{M}_{\mathbb{C}}$ the set of continuous multivalued functions with connected values from $X$ to $C L(X)$ and use capital letters for multifunctions and small ones for single valued functions.

Below we recall the well known uniform metric between members of $\mathbb{C}$ and the uniform Hausdorff metric between members of $\mathbb{M}$ :

$$
\begin{gathered}
\varrho(f, g)=\sup \{d(f(x), g(x)): x \in X\} \\
\varrho_{H}(F, G)=\sup \left\{d_{H}(F(x), G(x)): x \in X\right\} .
\end{gathered}
$$

A point $p$ of $X$ is a fixed point of $f$ iff $p=f(p)$, while it is fixed for $F$ iff $p \in F(p)$. The set of all fixed points of $f$ or $F$, that we denote by $F i x(f)$ or Fix $(F)$, is clearly closed. A fixed point $p$ of $f$ is called essential w.r.t. a family $\mathscr{C}$ of functions in $\mathbb{C}[4]$ iff for every neighborhood $U$ of $p$, there is a $\delta>0$ such that every $g$ in $\mathscr{C}$ with $\varrho(f, g)<\delta$ has a fixed point in $U$ (Cf. [3]). If every fixed point of $f$ is essential w.r.t. $\mathscr{C} f$ is said to be an essential map w.r.t. $\mathscr{C}$. A fixed point $p$ of $F$ is called essential w.r.t. a family $\mathscr{M}$ of multivalued continuous functions iff for every neighborhood $U$ of $p$, there is a $\delta>0$ such that every $G$ in $\mathscr{M}$ with $\varrho_{H}(F, G)<\delta$ has a fixed point in $U$. If every fixed point of $F$ is essential w.r.t. $\mathscr{M}, F$ is called an essential map w.r.t. $\mathscr{M}$.

M. K. Fort proved that if $p$ is the unique fixed point of a function it is essential and that the identity map has no essential fixed point. Also due to Fort is the following result.

Theorem 2.1 (Fort). Let $f$ belong to $\mathbb{C}$ and $p$ be a point of $X$. If $p$ has arbitrary small neighborhood $V$ such that $\bar{V}$ has the fixed point property and $f(\bar{V}) \subseteq V$, then $p$ is an essential fixed point of $f$.

We wish to point out that the condition of 2.1 is not necessary. Let us consider the function $f(x)=1-x$ from $[0,1]$ to $[0,1]$; it has only one fixed point $1 / 2$ which is essential, but for $0<\varepsilon<1 / 2$

$$
f([1 / 2-\varepsilon, 1 / 2+\varepsilon]) \nsubseteq(1 / 2-\varepsilon, 1 / 2+\varepsilon) .
$$

Let us prove the following result which is true for functions and multifunctions.

Theorem 2.2. The set of all essential fixed point of a function or a multifunction is closed. 
Proof. We prove the result for a multifunction; the one for a function is similar. Suppose $F$ is a multifunction, $p_{n} \in F\left(p_{n}\right)$ is essential and $\left(p_{n}\right)_{n}$ converges to $p$. If $U$ is a neighborhood of $p$, then eventually $p_{n}$ belongs to $U$ and there is a $\delta>0$ such that if $G$ is in $\mathscr{M}$ and $\varrho_{H}(F, G)<\delta, G$ has a fixed point in $U$.

Later on we give an example to show that it is possible for an essential fixed point to be a limit of a sequence of non essential fixed points (see Example 3.6.).

\section{Essential fixed points for functions.}

In this section we completely characterize essential fixed points of a function $f$ w.r.t. $\mathbb{C}$, if $X=[0,1]$. To simplify the statements we will not refer to $\mathbb{C}$. By the Brouwer [1] fixed point theorem, each $f$ has a fixed point.

Theorem 3.1. Let $p \in(0,1)$ be a fixed point for $f$. Then $p$ is an essential fixed point of $f$ iff every neighborhood of $p$ contains points $a$ and $b$ such that $f(a)>a$ and $f(b)<b$.

Proof. Let $\varepsilon>0$ be arbitrary but such that

$$
U=(p-\varepsilon, p+\varepsilon) \subseteq(0,1) .
$$

There are $a, b$ in $U$ such that $f(a)-a=\varepsilon_{1}>0$ and $b-f(b)=\varepsilon_{2}>0$. Set

$$
\delta=\frac{1}{2} \min \left\{\varepsilon, \varepsilon_{1}, \varepsilon_{2}\right\} .
$$

If $g$ satisfies the condition $\varrho(f, g)<\delta$, then $g(a)-a>\varepsilon_{1}-\delta>0$ and $g(b)-b<0$, therefore the function $g(x)-x$ must vanish between $a$ and $b$. So $g$ has a fixed point in $U$, showing that $p$ is essential for $f$. On the other hand, suppose that there exists a neighborhood $U$ of $p$ such that for each $x \in U$ either (i) $f(x) \leq x$ or (ii) $f(x) \geq x$. We need consider only (i). Since $f$ is continuous, there is a neighborhood $V$ of $p$ such that $f(V) \subset U$. Let $z$ and $y$ be in $V$ such that $z<p<y$, and let $\delta$ be arbitrary but less than 1. Set $g$ by

$$
g(x)=f(x)+(1-f(x)) d(x,\{z, y\}) \delta \quad \text { for } \quad x \in[z, y]
$$

and $g(x)=f(x)$ otherwise. Clearly $0 \leq g(x) \leq f(x)+(1-f(x)) \delta \leq 1$ for $x \in[0,1], \varrho(f, g)<\delta$ and $g$ has no fixed point in $[z, y]$. So $p$ is not essential.

Similar results concerning the end points of $[0,1]$ are given by the following corollary. 
Corollary 3.2. 0 is an essential fixed point of $f$ iff for each $\varepsilon>0$ there is an $x \in(0, \varepsilon)$ such that $f(x)<x .1$ is an essential fixed point of $f$ iff for each $\varepsilon>0$ there is an $x \in(1-\varepsilon, 1)$ such that $f(x)>x$.

We observe that if Fix $(f)$ contains a subset dense in an interval, then by Theorem 2.2 it contains an interval and by Theorem 3.1 no interior point of Fix $(f)$ can be essential. Therefore,

Corollary 3.3. If $f$ is an essential map, Fix $(f)$ cannot contain a subset dense in an interval.

If Fix $(f)$ contains an interval, the end points may be essential or not as we show with the following examples.

Example 3.1. Let $f(x)=x$ if $0 \leq x \leq 1 / 2$ and $f(x)=1 / 2$ otherwise. Here $\operatorname{Fix}(f)=[0,1 / 2]$ and both 0 and $1 / 2$ are not essential.

Example 3.2. Let $K=\left\{\frac{n+3}{2(n+1)}: n \in N\right\}$ and

$$
f(x)= \begin{cases}x & \text { if } \quad x \in[0,1 / 2] \\ x+(-1)^{n+1} d(x, K) & \text { if } \quad \frac{n+4}{2(n+2)} \leq x \leq \frac{n+3}{2(n+1)} .\end{cases}
$$

Each element of $K$ is essential by Theorem 3.1, and $1 / 2$ being a limit point of $K$ is essential by Theorem 2.2, while 0 is not essential.

The following result gives information about the cardinality of Fix $(f)$, when $F i x(f)$ is finite.

Theorem 3.4. Let $f$ be an essential map. If Fix $(f)$ is finite, then it must be odd.

Proof. First we note that a point $p$ at $(0,1)$ is essential iff the graph $G(f)$ of $f$ crosses the diagonal $\Delta$ in the point $(p, p)$. Suppose that only one of the end points is fixed, for example 0 . Then $f(0)=0, f(1)<1$ and there is an $\varepsilon>0$ such that $f(x)<x$ for each $x \in(0, \varepsilon)$. If 0 is the only fixed point of $f$, we are done. If not, $G(f)$ must cross $\Delta$ an even number of times in $(0,1)$. So Fix $(f)$ is odd. If both the end points are fixed, there exists an $\varepsilon>0$ such that $f(x)<x$ for each $x \in(0, \varepsilon)$ and $f(x)>x$ for each $x \in(1-\varepsilon, 1)$. So $G(f)$ must cross $\Delta$ an odd number of times and Fix $(f)$ is odd. If Fix $(f)$ does not contain the end points then $f(0)>0$ and $f(1)<1$. So $G(f)$ must cross $\Delta$ an odd number of times.

The above results show that if $f$ is an essential map, then Fix $(f)$ is made up of suitable combinations of the following basic cases:

(i) Fix $(f)$ is finite and contains an odd number of elements.

(ii) Fix $(f)$ is countably infinite but not dense in any interval. 
(iii) Fix $(f)$ is uncountable but does not contain any interval e.g. Cantor Set.

We now show a technique which enables us to construct essential maps illustrating the above cases.

Example 3.3. Let $K=\left\{a_{i}, 1 \leq i \leq 2 n+1\right\}$ be an odd number of points in $[0,1]$. We construct an essential map $f$ having $\operatorname{Fix}(f)=K$.

If $a_{1}=0$ and $a_{2 n+1}=1$ we put

$$
f(x)=x+(-1)^{i} d(x, K)
$$

If $a_{1}>0$ and $a_{2 n+1}=1$ we put

$$
f(x)=\left\{\begin{array}{lll}
a_{1} & \text { if } & 0 \leq x \leq a_{1} \\
x+(-i)^{i} d(x, K) & \text { if } & a_{i} \leq x \leq a_{i+1}
\end{array}\right.
$$

If $a_{1}>0$ and $a_{2 n+1}<1$ we put

$$
f(x)=\left\{\begin{array}{lll}
a_{1} & \text { if } & 0 \leq x \leq a_{1} \\
x+(-1)^{i} d(x, K) & \text { if } & a_{i} \leq x \leq a_{i+1} \\
a_{2 n+1} & \text { if } & a_{2 n+1} \leq x \leq 1
\end{array}\right.
$$

Example 3.4. Let $K$ be the set $\{1 / n, n \in N\} \cup\{0\}$. The function $f$ defined by

$$
f(x)= \begin{cases}0 & \text { if } \quad x=0 \\ x-(-1)^{n} d(x, K) & \text { if } \quad \frac{1}{n+1} \leq x \leq \frac{1}{n},\end{cases}
$$

is an essential map with $\operatorname{Fix}(f)=K$.

Example 3.5. Let $K$ be the standard Cantor set in $[0,1]$, which is obtained by removing $2^{n}$ middle third open intervals for each $n \in N$. We set $f(x)=x$ for each $x$ in $K$. If $x$ is in any one of the $2^{n}$ open intervals removed at the $n^{\text {th }}$ stage, we set

$$
f(x)=x+(-1)^{n} d(x, K)
$$

It can be verified that $f$ is an essential map with $F i x(f)=K$.

We observe that a sequence of non-essential fixed points of $f$ may converge to an essential fixed point or to a non essential one.

Example 3.6. Let $K$ be the set $\{1 / n, n \in N\} \cup\{0\}$ and $f$ the function defined by $f(x)=x-d(x, K)$. Each point $p=1 / n$ is non essential but 0 is essential. For the function $g$ defined by $g(x)=x+d(x, K)$ each point $p=\frac{1}{n+1}$ is non essential as is 0 . 
S. Kinoshita [8] first introduced the concept of an essential component of a continuous function. A component $H \subset F i x(f)$ is essential iff for every neighborhood $U$ of $H$, there is a $\delta>0$ such that every $g$ in $\mathscr{C}$ with $\varrho(f, g)<$ $\delta$ has a fixed point in $U$. Obviously it is possible for a function have no essential fixed point and yet have an essential component, see for example $f(x)=x$ for $x$ in $[0,1]$. It is easy to see that if $p \in H$ is an essential fixed point of $f$, then $H$ is obviously an essential component of $f$. Therefore we will consider only components which are closed intervals containing no essential fixed point. Let $f$ be a continuous function in $[0,1]$ and $[c, d]$ a typical component contained in Fix $(f)$. If $c=0$ (resp. $d=1$ ), then $c$ (resp. $d)$ is not an essential fixed point. If $c=0$ and $d=1$ we have already seen that it is an essential component. In the following we assume that no $x \in[c, d]$ is essential.

Similar to Theorem 3.1 and Corollary 3.2 we have the following results:

Proposition 3.5. $[c, d] \subset F i x(f) \cap(0,1)$ is an essential component iff every neighborhood of $[c, d]$ contains points $a$ and $b$ such that $f(x)-x$ has opposite signs at $a$ and $b$.

Proposition 3.6. If $d<1,[0, d] \subset$ Fix $(f)$ is an essential component of $f$ iff every neighborhood of $d$ contains a point $x$ with $f(x)<x$.

Proposition 3.7. If $c>0,[c, 1] \subset F i x(f)$ is an essential component iff for each $\varepsilon>0$ there is a point $x \in(c-\varepsilon, c)$ with $f(x)>x$.

A simple example of a typical essential component is the following: $[0,1 / 2]$ is an essential component for the function $f(x)=x$ if $x \in[0,1 / 2], f(x)=1 / 2$ otherwise.

\section{Essential fixed points of continuous multifunctions.}

In this section we study essential fixed points of continuous multifunctions $F$. We show that some of the results are quite different from those of continuous single valued functions while others are similar. Strother ([11], [12]) has shown that in the space $[0,1]$ each $F \in \mathbb{M}$ has a fixed point whereas its analogue for the unit square is not true. In Theorem 3.4 we showed that if $F i x(f)$ is finite for an essential map, then it must be odd. In contrast to this we give examples of essential multifunctions having an even (Example 4.1) as well an odd (Example 4.2) number of fixed points.

Example 4.1. Let $F$ be the multifunction defined by $F(x)=\left\{x^{2}, \sqrt{x}\right\}$.In this case $F i x(F)=\{0,1\}$ and each of 0,1 is an essential fixed point of $F$ w.r.t. $\mathbb{M}$. 
Example 4.2. Let $K=\{0,1,1 / 2\}$ and the multifunction $G$ defined by

$$
G(x)=\left\{\begin{array}{lll}
\{x+d(x, K), 0,1\} & \text { if } & 0 \leq x \leq 1 / 2 \\
\{x-d(x, K), 0,1\} & \text { if } & 1 / 2 \leq x \leq 1
\end{array}\right.
$$

$G$ has three fixed points and each is essential, in contrast to the previous one in which there are only two.

In Theorem 3.1 we showed that for a single valued function $f$, a fixed point $p$ is essential iff in each nbhd of $p, f$ is not unisigned. In contrast to this we have the following example for multifunctions.

Example 4.3. Let $F$ be the multifunction $F(x)=\left\{x^{2},[x, \sqrt{x}]\right\}$. In this case $\operatorname{Fix}(F)=[0,1], 0$ and 1 are essential w.r.t. $\mathbb{M}$, but there are points satisfying a condition similar to Theorem 3.1 which are not essential. Choose $p=1 / 2 \quad \varepsilon=1 / 16$ and $x_{1}, x_{2}$ in $(p-\varepsilon, p+\varepsilon)$ such that $x_{1}<p<x_{2}$ with $y_{i} \in F\left(x_{i}\right)$ satisfying $y_{1}<x_{1}$ and $y_{2}>x_{2}$. Let $\delta>0$ be less than $1 / 2 \min \left\{\varepsilon, x_{1}-y_{1}, y_{2}-x_{2}\right\}$ and satisfying $p+\delta<1$. Set

$$
G(x)= \begin{cases}\left\{x^{2}, y+\delta(1-y) d\left(x,\left\{x_{1}, x_{2}\right\}\right): y \in[x, \sqrt{x}]\right\} & \text { if } x_{1} \leq x \leq x_{2} \\ F(x) & \text { otherwise }\end{cases}
$$

It can be verified that $G$ belongs to $\mathbb{M}, \varrho_{H}(F, G)<\delta$ and $G$ has no fixed point in $\left(x_{1}, x_{2}\right)$.

For elements of $\mathbb{M}$ we can prove only the analogue of a part of Theorem 3.1 .

Theorem 4.1. Let $p \in(0,1)$ be a fixed point for $F \in \mathbb{M}$. If there exists a neighborhood $U$ of $p$ such that for each $x$ in $U$ and for each $y$ in $F(x)$ $x \leq y \quad($ or $y \leq x)$, then $p$ is not an essential fixed point of $F$.

Proof. We give an outline in the case $x \leq y$. Let $x_{1}$ and $x_{2}$ be points of $U$ satisfying $x_{1} \leq p \leq x_{2}$. Let $\delta>0$ such that for each $x \in\left[x_{1}, x_{2}\right] \quad x+\delta<1$. The multifunction $G$ defined by

$$
G(x)= \begin{cases}\left\{y+\delta(1-y) d\left(x,\left\{x_{1}, x_{2}\right\}\right): y \in F(x)\right\} & \text { if } x_{1} \leq x \leq x_{2} \\ F(x) & \text { otherwise }\end{cases}
$$

belongs to $\mathbb{M}$, has no fixed point in $\left(x_{1}, x_{2}\right)$ and $\varrho_{H}(F, G)<\delta$.

With the same technique it is possible to obtain conditions assuring that 0 or 1 aren't essential fixed points.

We can obtain results similar to those of functions if we consider multifunctions with connected values. We can prove the following result: 
Theorem 4.2. Suppose that $F \in \mathbb{M}_{\mathbb{C}}$, and $p \in(0,1) \cap F i x(F)$. If each neighborhood of $p$ contains $x_{1}, x_{2}$ such that there are $y_{i} \in F\left(x_{i}\right)$ satisfying $y_{1}<x_{1}$ and $y_{2}>x_{2}$, then $p$ is an essential fixed point of $F$ w.r.t. $\mathbb{M}_{\mathbb{C}}$

Proof. We give an outline. Using the definition of the Hausdorff metric $d_{H}$ it follows that if $\mathrm{G}$ belongs to $\mathbb{M}_{\mathbb{C}}$ and $d_{H}(F, G)<\delta$ for sufficiently small positive $\delta$ there exist $z_{i} \in G\left(x_{i}\right)$ satisfying $z_{1}<x_{1}$ and $z_{2}>x_{2}$. Since $G\left(\left[x_{1}, x_{2}\right]\right)$ is connected there is a point $x_{o}$ in $\left(x_{1}, x_{2}\right) \cap G\left(x_{o}\right)$.

For multifunctions with connected values it is possible to obtain a result for the end-points.

Theorem 4.3. 0 is an essential fixed point of $F \in \mathbb{M}_{\mathbb{C}}$ w.r.t. $\mathbb{M}_{\mathbb{C}}$ if each neighborhood of 0 contains points $b<a$ and $b \in F(a)$. A similar result is true for 1 .

We observe that for every compact and connected subset $K$ of $[0,1]$ the multifunction $F(x)=K$ is in $\mathbb{M}_{\mathbb{C}}$ and each element of $K$ is essential.

\section{Selections and essential fixed points.}

If $F$ is a multifunction on $X$ then a selection for $F$ is a function $f$ such that for each $x \in X, f(x) \in F(x)$. Strother [11] has shown that each continuous multifunction $F$ has continuous selections given by $f(x)=$ l.u.b.F $F(x)$ and $g(x)=g . l . b . F(x)$. In this section we study the relationships between the essential fixed points of $F$ and those of their selections. Simple examples prove that "essentiality" does not pass from a multifunction to its selections or viceversa.

Example 5.1 Let us consider in $[0,1] \quad F(x)=\left\{\sqrt{x}, x^{2}\right\}$ and $f(x)=x^{2}$. 1 is not essential for $f$ w.r.t. $\mathbb{C}$ but is essential for $F$ w.r.t. $\mathbb{M}$.

Example 5.2 Let us consider in $[0,1] \quad K=\{0,1,1 / 2\}$ and the functions:

$$
\begin{aligned}
& f(x)=\left\{\begin{array}{lll}
x+d(x, K) & \text { if } & \frac{1}{2} \leq x \leq 1 \\
x-d(x, K) & \text { if } & 0 \leq x<\frac{1}{2}
\end{array}\right. \\
& g(x)=\left\{\begin{array}{lll}
x-d(x, K) & \text { if } & \frac{1}{2} \leq x \leq 1 \\
x+d(x, K) & \text { if } & 0 \leq x<\frac{1}{2}
\end{array}\right.
\end{aligned}
$$

It is easy to prove that $1 / 2$ is essential for both $f$ and $g$ but it is not essential for the multifunction $F(x)=\{f(x), g(x)\}$. Choose $x_{1}, x_{2}$ in $[0,1]$ such that $x_{1},<\frac{1}{2}<x_{2}$ and $x_{2}-x_{1}<\frac{1}{8}$ and $\delta$ satisfying $0<\delta<\frac{1}{8}$. The 
multifunction

$$
G(x)=\left\{\begin{array}{cc}
\left\{x+d(x, K)+\delta d\left(x,\left\{x_{1}, x_{2}\right\}\right),\right. & \\
\left.x-d(x, K)-\delta d\left(x,\left\{x_{1}, x_{2}\right\}\right)\right\} & \text { if } x_{1} \leq x \leq x_{2} \\
F(x) & \text { otherwise }
\end{array}\right.
$$

belongs to $\mathbb{M}$, satisfies the condition $\varrho_{H}(F, G)<\delta$ but has no fixed point in $\left(x_{1}, x_{2}\right)$.

We observe that for multifunctions with connected values we have the following result whose proof follows from Theorems 3.1 and 4.3 .

Proposition 5.1. Let $F$ be in $\mathbb{M}_{\mathbb{C}}$ and $f$ be a continuous selection of $F$. If $p$ is an essential fixed point for $f$ w.r.t. $\mathbb{C}$, then $p$ is an essential fixed point of $F$ w.r.t. $\mathbb{M}_{\mathbb{C}}$.

In general the converse is false, for example consider the multifunction $F(x)=[0,1]$ and as selection $f(x)=x$.

The situation is different in the case of contractive multifunctions. that is multifunctions satisfying $d_{H}(F(x), F(y))<d(x, y)$ for every $x, y$. It is well known that each contractive function has a unique fixed point (and hence is an essential map) while a contractive multifunction may have many fixed points. On the other hand if $X=[0,1]$ a multifunction with connected values $F(x)=[f(x), g(x)]$ is contractive iff each $f$ and $g$ are contractive. We prove the following result:

Theorem 5.2. If $F$ belongs to $\mathbb{M}_{\mathbb{C}}$ and is contractive, then $F$ is an essential map w.r.t. $\mathbb{M}_{\mathbb{C}}$.

Proof. Let $F(x)=[f(x), g(x)]$ and $p$ fixed for $F$. There is a continuous selection $h(x)$ for which $p$ is essential. Indeed, since $p$ belongs to $[f(p), g(p)]$ there are $\alpha, \beta$ in $[0,1]$ for which $p=\alpha f(p)+\beta g(p)$, and the function $h(x)=\alpha f(x)+\beta g(x)$ is a contractive selection. Therefore $p$ is an essential fixed point of $F$.

\section{Essential fixed points of non expansive functions be- tween Banach spaces.}

If $X$ is a Banach space, a mapping $g$ on $X$ to $X$ is called non expansive provided $\|g(x)-g(y)\| \leq\|x-y\|$ for all $x, y$ in $X$. For mappings of this type there need not be any fixed points nor need a fixed point be unique if it does exist. In [2] Browder has proved that: 
Theorem 6.1 (Browder). Let $X$ be a uniformly convex Banach space, $g$ a non expansive mapping on $X$ to $X$ and $B$ a non empty bounded convex closed subset of $X$ for which $g(B) \subset B$. Then the set of the fixed points of $g$ in $B$ is non empty closed and convex.

Under the same hypotheses of Browder's theorem we prove that:

Theorem 6.2. If there exist in $B$ more than one fixed point, none of them is essential.

Proof. Let $p$ and $p^{\prime}$ be fixed points of $g$ in $B$ and let $U(p)$ be a neighborhood of $p$ which does not contain $p^{\prime}$. The sequence

$$
g_{n}(x)=\frac{p^{\prime}}{n}+\left(1-\frac{1}{n}\right) g(x), \quad x \in X
$$

converges uniformly to $g(x)$ in $B$ since

$$
\left\|g_{n}(x)-g(x)\right\|=\frac{1}{n}\left\|p^{\prime}-g(x)\right\| \leq \frac{1}{n} \sup _{x \in C}\left\|p^{\prime}-g(x)\right\| .
$$

$g_{n}$ is a contraction having $p^{\prime}$ as unique fixed point and $p^{\prime}$ does not belong to $U(p)$. Now we prove that there exists a mapping $g^{\prime}$ on $X$ to $X$ satisfying $g^{\prime}(B) \subset B$ and sufficiently near to $g$ which has no fixed point in $U(p)$. If $B^{\prime}$ is a bounded subset of $X$ containing $B$ let us consider, for $\delta>0$ and $g_{m}$ satisfying the condition $\sup _{x \in B}\left\|g(x)-g_{m}(x)\right\|<\delta$

$$
g^{\prime}(x)=\left\{\begin{array}{lll}
g(x) & \text { if } \quad x \notin B^{\prime} \\
g_{m}(x) & \text { if } x \in B \\
g_{m}(x) \alpha(x)+g(x) \beta(x) & \text { if } \quad x \in B^{\prime}-B
\end{array}\right.
$$

where

$$
\alpha(x)=\frac{d\left(x, \partial B^{\prime}\right)}{d\left(x, \partial B^{\prime}\right)+d(x, \partial B)} \quad \text { and } \quad \beta(x)=\frac{d(x, \partial B)}{d\left(x, \partial B^{\prime}\right)+d(x, \partial B)} .
$$

$g^{\prime}$ is continuous in $X$ and for every $x$ in $X$ satisfies the condition $\| g^{\prime}(x)-$ $g(x) \|<\delta$.

We observe that if we fix $p, U(p)$ and $p^{\prime} \notin U(p)$, the same sequence

$$
g_{n}(x)=\frac{p^{\prime}}{n}+\left(1-\frac{1}{n}\right) g(x), \quad x \in X
$$

can be used to prove that every fixed point in $U(p)$ is not essential. See the following example [13]. Let $X$ be the space $R^{2}$ with the Euclidean norm and let $B$ be the set

$$
\left\{(r, \vartheta), 0 \leq r \leq 1, \frac{-\pi}{2} \leq \vartheta \leq \frac{-\pi}{4}\right\}
$$


where $(r, \vartheta)$ denotes polar coordinates. If we define in $B$ the mapping $g(r, \vartheta)=(r,-\pi / 2)$ we obtain a non expansive mapping whose set of fixed points is the line segment $F=\{(r,-\pi / 2), 0 \leq r \leq 1$,$\} . Using the approx-$ imating sequence $g_{n}(r, \vartheta)=((1-1 / n) r,-\pi / 2)$ whose unique fixed point is $(0,-\pi / 2)$ we can prove that every point $(r,-\pi / 2)$ with $1 / 2 \leq r$ is not essential. Using the approximating sequence

$$
g_{n}^{\prime}(r, \vartheta)=\left(\left(1+\frac{1-r}{n}\right) r,-\frac{\pi}{2}\right)
$$

whose unique fixed point is $(1,-\pi / 2)$ we can prove that every point $(r,-\pi / 2)$ with $1 / 2<r$ is not essential.

For mappings between Banach spaces, that are non necessarily non expansive we can prove the following:

Theorem 6.3. Let $X$ be a Banach space with fixed point property and $g$ a mapping from $X$ to $X$. If Fix(g) has interior points, none of them is essential.

Proof. Let $p$ be interior to Fix $(g)$ and $U(p)$ be a bounded open neighborhood of $p$ contained in Fix $(g)$. If $w_{0}$ is a point of $X$ and $\left|w_{0}\right| \neq 0$ we define

$$
g_{n}(x)=\left\{\begin{array}{lll}
g(x) & \text { if } & x \notin U(p) \\
g(x)+\frac{d(x, \partial U(p))}{n \delta(U(p))} w_{0} & \text { if } & x \in U(p),
\end{array}\right.
$$

where $\delta(U(p))$ is the diameter of $U(p)$ and $\partial U(p)$ its boundary. $g_{n}$ is continuous, has no fixed point in $U(p)$ since in $U(p)$

$$
\left\|g_{n}(x)-x\right\|=\left\|g(x)-x+\frac{d(x, \partial U(p))}{n \delta(U(p))} w_{0}\right\|=\left\|w_{0}\right\| \frac{d(x, \partial U(p))}{n \delta(U(p))} .
$$

Since $\left\|g_{n}(x)-g(x)\right\| \leq \frac{\left\|w_{0}\right\|}{n}$, the sequence $g_{n}(x)$ converges uniformly to $g(x)$.

\section{References}

[1] L. E. J. Brouwer, Uber Abbildungen von Mannigfaltigkeiten, Math. Ann. 71(1912), 97-115.

[2] F. E. Browder, Convergence of approximants to fixed points of non expansive non linear mappings in Banach spaces, Arch. Rat. Mech. Anal. 24(1967a), 82-90. 
[3] A. M. Bruckner, Stability in the family of $\omega$-limit sets of continuous self-maps of the interval, Real Analysis Exchange, 22(1997), 52-75.

[4] M. K. Fort, Jr, Essential and non essential fixed points, Amer. J. Math. 72(1950), 315-322.

[5] G. Gabor, On the classification of fixed points, Math. Japonica, v.40, n.2, (1994), 361-369.

[6] Jiang, Jia-He, Essential fixed points of the multivalued mappings, Sci. Sinica, v.11, n.3, (1962), 293-298.

[7] Jiang, Jia-he, Essential component of the set of fixed points of the multivalued mappings and its application to the theory of games, Sci. Sinica, v.12, n.7, (1963), 951-964.

[8] S. Kinoshita, On essential components of the set of fixed points, Osaka Math. J. 4(1952), 19-22.

[9] T. Kok-Keong, Y. Jian, Y. Xian-Zhi, The stability of coincident points for multivalued mappings, Nonlinear Analysis, Theory, Methods and Applications, vol. 25, No. 2, (1995) 163-168.

[10] B. O’Neill. Essential sets and fixed points, Amer. J. Math. 75(1953), 497-509.

[11] W. Strother, On an open question concerning fixed points, Proc. Amer. Math. Soc. 4(1953), 988-993.

[12] W. Strother, Fixed points, fixed sets, and M-retracts, Duke. Math. J. 22(1955), 551-556.

[13] H. F. Senter, W. J. Dotson, Approximating fixed points of non expansive mappings, Proc. Amer. Math. Soc. 44(1974), 375-390.

[14] Y. Yonezawa, On f.p.p and $f^{*} \cdot p . p$ of some not locally connected continua, Fund. Math. 139(1991), 91-98. 
